\title{
Notification List Notification that new names and new combinations
have appeared in volume 58, part 11 , of the IJSEM
}

Correspondence

Jean Euzéby

email address can be found at

www.bacterio.net
This listing of names published in a previous issue of the IJSEM is provided as a service to bacteriology to assist in the recognition of new names and new combinations. This procedure was proposed by the Judicial Commission [Minute 11(ii), Int J Syst Bacterio/ 41 (1991), p. 185]. The names given herein are listed according to the Rules of priority (i.e. page number and order of valid publication of names in the original articles). Taxonomic opinions included in this List (i.e. the creation of synonyms or the emendation of circumscriptions) cannot be considered as validly published nor, in any other way, approved by the International Committee on Systematics of Prokaryotes and its Judicial Commission.

\begin{tabular}{|c|c|c|}
\hline Name/author(s): & Proposed as: & Page no. \\
\hline Pseudonocardia carboxydivorans Park et al. 2008 & sp. nov. & 2477 \\
\hline Pseudonocardia Henssen 1957 (Approved Lists 1980) emend. Park et al. 2008 & emend.* & 2477 \\
\hline Melitea salexigens Urios et al. 2008 & sp. nov. & 2482 \\
\hline $\begin{array}{l}\text { Rhizobium trifolii Dangeard } 1926 \text { (Approved Lists 1980) pro synon. Rhizobium leguminosarum } \\
\text { (Frank 1879) Frank } 1889 \text { (Approved Lists 1980) }\end{array}$ & synon. ${ }^{*}$ & 2487 \\
\hline $\begin{array}{l}\text { Rhizobium leguminosarum (Frank 1879) Frank } 1889 \text { (Approved Lists 1980) emend. } \\
\text { Ramírez-Bahena et al. } 2008\end{array}$ & emend.* & 2489 \\
\hline Rhizobium phaseoli Dangeard 1926 (Approved Lists 1980) emend. Ramírez-Bahena et al. 2008 & emend. ${ }^{*}$ & 2489 \\
\hline Haloferula Yoon et al. 2008 & gen. nov. & 2495 \\
\hline Haloferula rosea Yoon et al. 2008 & sp. nov. & 2495 \\
\hline Haloferula harenae Yoon et al. 2008 & sp. nov. & 2497 \\
\hline Salinimicrobium terrae Chen et al. 2008 & sp. nov. & 2503 \\
\hline Croceitalea Lee et al. 2008 & gen. nov. & 2509 \\
\hline Croceitalea eckloniae Lee et al. 2008 & sp. nov. & 2509 \\
\hline Croceitalea dokdonensis Lee et al. 2008 & sp. nov. & 2509 \\
\hline Hellea Alain et al. 2008 & gen. nov. & 2516 \\
\hline Hellea balneolensis Alain et al. 2008 & sp. nov. & 2516 \\
\hline Nocardiopsis arabia Hozzein and Goodfellow 2008 & sp. nov. & 2523 \\
\hline Glycomyces endophyticus Qin et al. 2008 & sp. nov. & 2527 \\
\hline Desulfotomaculum hydrothermale Haouari et al. 2008 & sp. nov. & 2533 \\
\hline Microbacterium kribbense Dastager et al. 2008 & sp. nov. & 2539 \\
\hline Thermodesulfovibrio aggregans Sekiguchi et al. 2008 & sp. nov. & 2547 \\
\hline Leucobacter tardus Behrendt et al. 2008 & sp. nov. & 2577 \\
\hline Photorhabdus temperata subsp. cinerea Tóth and Lakatos $2008 \dagger$ & subsp. nov. & 2580 \\
\hline Halomonas korlensis $\mathrm{Li}$ et al. 2008 & sp. nov. & 2586 \\
\hline
\end{tabular}


cont.

\begin{tabular}{|c|c|c|}
\hline Name/author(s): & Proposed as: & Page no. \\
\hline Alteromonas genovensis Vandecandelaere et al. 2008 & sp. nov. & 2593 \\
\hline $\begin{array}{l}\text { Alteromonas macleodii Baumann et al. } 1972 \text { (Approved Lists 1980) emend. Vandecandelaere } \\
\text { et al. } 2008\end{array}$ & emend.* & 2594 \\
\hline Acanthopleuribacter Fukunaga et al. 2008 & gen. nov. & 2600 \\
\hline Acanthopleuribacter pedis Fukunaga et al. 2008 & sp. nov. & 2600 \\
\hline Acanthopleuribacteraceae Fukunaga et al. 2008 & fam. nov. & 2600 \\
\hline Acanthopleuribacterales Fukunaga et al. 2008 & ord. nov. & 2600 \\
\hline Holophagaceae Fukunaga et al. 2008 & fam. nov. & 2600 \\
\hline Holophagales Fukunaga et al. 2008 & ord. nov. & 2600 \\
\hline Holophagae Fukunaga et al. 2008 & class. nov. & 2601 \\
\hline Streptomyces specialis Kämpfer et al. 2008 & sp. nov. & 2605 \\
\hline $\begin{array}{l}\text { Myxococcus flavescens Yamanaka et al. } 1990 \text { pro synon Myxococcus virescens Thaxter } 1892 \text { (Approved } \\
\text { Lists 1980) }\end{array}$ & synon.* & 2608 \\
\hline Myxococcus virescens Thaxter 1892 (Approved Lists 1980) emend. Lang et al. 2008 & emend.* & 2608 \\
\hline Mesorhizobium gobiense Han et al. 2008 & sp. nov. & 2616 \\
\hline Mesorhizobium tarimense Han et al. 2008 & sp. nov. & 2616 \\
\hline Nocardioides dokdonensis Park et al. 2008 & sp. nov. & 2622 \\
\hline Kriegella Nedashkovskaya et al. 2008 & gen. nov. & 2625 \\
\hline Kriegella aquimaris Nedashkovskaya et al. 2008 & sp. nov. & 2627 \\
\hline Bacillus alkalitelluris Lee et al. 2008 & sp. nov. & 2632 \\
\hline Chryseobacterium oranimense Hantsis-Zacharov et al. 2008 & sp. nov. & 2638 \\
\hline Paenibacillus taichungensis Lee et al. 2008 & sp. nov. & 2643 \\
\hline Mesorhizobium caraganae Guan et al. 2008 & sp. nov. & 2650 \\
\hline Thiophaeococcus Anil Kumar et al. 2008 & gen. nov. & 2663 \\
\hline Thiophaeococcus mangrovi Anil Kumar et al. 2008 & sp. nov. & 2663 \\
\hline
\end{tabular}

${ }^{\star}$ Taxonomic opinion.

$\dagger$ According to Rule 40d (formerly Rule 46) of the Bacteriological Code (1990 Revision), the valid publication of Photorhabdus temperata subsp. cinerea Tóth and Lakatos 2008 automatically creates another subspecies, Photorhabdus temperata subsp. temperata Fischer-Le Saux et al. 1999. 